\title{
THE ASYMPTOTIC DISTRIBUTION OF ANDREWS' SMALLEST PARTS FUNCTION
}

\author{
JOSIAH BANKS, ADRIAN BARQUERO-SANCHEZ, RIAD MASRI, YAN SHENG
}

\begin{abstract}
In this paper, we use methods from the spectral theory of automorphic forms to give an asymptotic formula with a power saving error term for Andrews' smallest parts function $\operatorname{spt}(n)$. We use this formula to deduce an asymptotic formula with a power saving error term for the number of 2-marked Durfee symbols associated to partitions of $n$. Our method requires that we count the number of Heegner points of discriminant $-D<0$ and level $N$ inside an "expanding" rectangle contained in a fundamental domain for $\Gamma_{0}(N)$.
\end{abstract}

\section{INTRODUCTION AND STATEMENT OF RESULTS}

1.1. Overview. In [A2], Andrews defined the smallest parts function $\operatorname{spt}(n)$, which counts the number of smallest parts associated to partitions of a positive integer $n$. For example, $\operatorname{spt}(4)=10$, which can be seen by considering the partitions of $n=4$ :

$$
\underline{4}, \quad 3+\underline{1}, \quad \underline{2}+\underline{2}, \quad 2+\underline{1}+\underline{1}, \quad \underline{1}+\underline{1}+\underline{1}+\underline{1} .
$$

The function $\operatorname{spt}(n)$ has many remarkable properties. Perhaps most notably, $\operatorname{spt}(n)$ satisfies congruences similar to Ramanujan's congruences for the partition function $p(n)$. For some examples of results in this direction, see the works [A2, ABL, FO, G, O].

Bringmann [B] gave an asymptotic formula for $\operatorname{spt}(n)$ in her work on partition statistics. In this paper, we will use methods from the spectral theory of automorphic forms to give an asymptotic formula for $\operatorname{spt}(n)$ with a power saving error term (see Theorem 1.3). An important input is recent work of Ahlgren and Andersen [AA] expressing $\operatorname{spt}(n)$ as the trace of a weak Maass form of weight zero for $\Gamma_{0}(6)$ along a Galois orbit of Heegner points of discriminant $-24 n+1$ and level 6 (see (1.3)). Their expression for $\operatorname{spt}(n)$ is analogous to a formula of Bruinier and Ono [BO] for $p(n)$.

Using work of Andrews [A, A2] which relates $\operatorname{spt}(n)$ to rank moments and marked Durfee symbols, we will also give an asymptotic formula with a power saving error term for the number of 2-marked Durfee symbols associated to partitions of $n$ (see Theorem 1.6).

A large portion of this paper is devoted to counting the number of Heegner points inside an "expanding" rectangle contained in a fundamental domain for $\Gamma_{0}(N)$ (see Proposition 3.2). This result is needed for the proofs of our main theorems, though it is of independent interest.

1.2. Preliminaries. To state our main results, we fix the following notation and assumptions. Let $N \geq 1$ be a squarefree integer and $-D<-4$ be an odd fundamental discriminant coprime to $N$ such that every prime divisor of $N$ splits in $K:=\mathbb{Q}(\sqrt{-D})$. Fix a solution $h$ $(\bmod 2 N)$ of $h^{2} \equiv-D(\bmod 4 N)$. Given a primitive integral ideal $\mathfrak{A}$ of $K$, one can write

$$
\mathfrak{A}=\mathbb{Z} a+\mathbb{Z} \frac{b+\sqrt{-D}}{2}, \quad a=N(\mathfrak{A}), \quad b=b_{\mathfrak{A}}^{(h)} \in \mathbb{Z}
$$


with $b \equiv h(\bmod 2 N)$ and $b^{2} \equiv-D(\bmod 4 N a)$. Here $N(\mathfrak{A})$ denotes the norm of $\mathfrak{A}$. Then

$$
\tau_{\mathfrak{A}}^{(h)}:=\frac{b+\sqrt{-D}}{2 N a}
$$

defines a Heegner point on $Y_{0}(N):=\Gamma_{0}(N) \backslash \mathbb{H}$. It is known that $\tau_{\mathfrak{A}}^{(h)} \in Y_{0}(N)$ depends only on the ideal class of $\mathfrak{A}$ and on $h(\bmod 2 N)$, so we denote it by $\tau_{[\mathfrak{a}]}^{(\hat{h})}$. For details concerning these facts, see [GZ, Part II, Section 1].

Let CL $(K)$ be the ideal class group of $K$ and $h(-D)$ be the class number. By Minkowski's theorem, we may choose a primitive integral ideal $\mathfrak{A}$ in each ideal class $[\mathfrak{A}] \in \mathrm{CL}(K)$ such that

$$
N(\mathfrak{A}) \leq \frac{2}{\pi} \sqrt{D}
$$

Having fixed such a choice $\mathfrak{A}$ for each ideal class, we define the Galois orbit

$$
\mathcal{O}_{D, N, h}:=\left\{\tau_{[\mathfrak{A}]}^{(h)}:[\mathfrak{A}] \in \mathrm{CL}(K)\right\}
$$

The set of all Heegner points of discriminant $-D$ and level $N$ is then given by

$$
\Lambda_{D, N}:=\bigcup_{\substack{h \\ h^{2} \equiv-D(\bmod 2 N)}} \mathcal{O}_{D, N, h} .
$$

Let $F: \mathbb{H} \rightarrow \mathbb{C}$ be a $\Gamma_{0}(N)$-invariant function. We define the "trace" of $F$ by

$$
\operatorname{Tr}_{D, N, h}(F):=\sum_{\tau \in \mathcal{O}_{D, N, h}} F(\tau)
$$

Next, we define the regularized integral of a weak Maass form of weight zero for $\Gamma_{0}(N)$. Let $\mathcal{F}$ be the standard fundamental domain for $\mathrm{SL}_{2}(\mathbb{Z})$. For $Y>0$, define the set

$$
\mathcal{F}(Y):=\{z \in \mathcal{F}: \operatorname{Im}(z) \leq Y\}
$$

Then if $\mathfrak{a}$ is a cusp of $\Gamma_{0}(N)$ and $\sigma_{\mathfrak{a}} \in \mathrm{SL}_{2}(\mathbb{R})$ is a scaling matrix such that $\sigma_{\mathfrak{a}}(\infty)=\mathfrak{a}$ (see e.g. $[\mathrm{I} 2,(2.15)$ and $(2.28)-(2.31)])$, we define the set

$$
\mathcal{F}_{N}(Y):=\bigcup_{\mathfrak{a}} \sigma_{\mathfrak{a}}(\mathcal{F}(Y))
$$

Let $M_{k}^{!}(N)$ (resp. $\left.W_{k}(N)\right)$ denote the space of weakly holomorphic (resp. weak Maass) forms of weight $k$ for $\Gamma_{0}(N)$. Given a weak Maass form $F \in W_{0}(N)$, we define the regularized integral

$$
\langle F, 1\rangle_{\mathrm{reg}}:=\lim _{Y \rightarrow \infty} \int_{\mathcal{F}_{N}(Y)} F(x+i y) \frac{d x d y}{y^{2}} \frac{1}{\operatorname{vol}\left(Y_{0}(N)\right)},
$$

provided the limit exists. Note that this limit exists for weakly holomorphic forms $F \in$ $M_{0}^{!}(N) \subset W_{0}(N)$ (see [M2, Proposition 6.1]). 
1.3. Asymptotics for $\operatorname{spt}(n)$. Consider the weakly holomorphic form

$$
f(z):=\frac{1}{2} \frac{E_{2}(z)-2 E_{2}(2 z)-3 E_{2}(3 z)+6 E_{2}(6 z)}{(\eta(z) \eta(2 z) \eta(3 z) \eta(6 z))^{2}} \in M_{-2}^{!}(6),
$$

where $E_{2}(z)$ is the usual weight 2 quasi-modular Eisenstein series and $\eta(z)$ is the Dedekind eta function. Define the Maass weight raising operator

$$
\partial:=\frac{1}{4 \pi}\left(2 i \frac{\partial}{\partial z}-2 \operatorname{Im}(z)^{-1}\right): M_{-2}^{!}(N) \longrightarrow W_{0}(N) .
$$

The image of $f(z)$ under the operator $\partial$ is a weak Maass form in $W_{0}(6)$ which we denote by $P(z)$. Bruinier and Ono $[\mathrm{BO}]$ proved the following formula expressing the partition function $p(n)$ as a trace of the weak Maass form $P(z)$,

$$
p(n)=\frac{1}{24 n-1} \operatorname{Tr}_{24 n-1,6,1}(P) .
$$

Let $e(z):=e^{2 \pi i z}$. The third author [M, Theorem 1.6] combined (1.1) with methods from the spectral theory of automorphic forms to prove the following asymptotic formula for $p(n)$.

Theorem $1.1\left([\mathrm{M}]\right.$, Theorem 1.6). Let $n \in \mathbb{Z}^{+}$be such that $24 n-1$ is squarefree. Then

$$
p(n)=\frac{1}{24 n-1} \sum_{\tau \in R_{24 n-1,6,1}^{*}}\left(1-\frac{1}{2 \pi \operatorname{Im}(\tau)}\right) e(-\tau)+\frac{h(-24 n+1)}{24 n-1}\langle P, 1\rangle_{\mathrm{reg}}+O_{\varepsilon}\left(n^{-\frac{89}{176}+\varepsilon}\right),
$$

where

$$
R_{24 n-1,6,1}^{*}:=\left\{\tau \in \mathcal{O}_{24 n-1,6,1}: \operatorname{Im}(\tau)>\frac{24}{\pi}+(24 n-1)^{-\frac{1}{176}}\right\} .
$$

Remark 1.2. Starting with a different formula for $p(n)$ due to Bringmann and Ono [BrO], Folsom and the third author $[\mathrm{FM}]$ used spectral methods to give an asymptotic formula for $p(n)$. Theorem 1.1 can in some respects be viewed as a refinement of that result.

Ahlgren and Andersen recently proved a formula analogous to (1.1) for Andrews' smallest parts function $\operatorname{spt}(n)$. Consider the weakly holomorphic form

$$
g(z):=\frac{1}{24} \frac{E_{4}(z)-4 E_{4}(2 z)-9 E_{4}(3 z)+36 E_{4}(6 z)}{(\eta(z) \eta(2 z) \eta(3 z) \eta(6 z))^{2}} \in M_{0}^{!}(6),
$$

where $E_{4}(z)$ is the usual weight 4 Eisenstein series. Ahlgren and Andersen [AA, Theorem 2] proved that

$$
\operatorname{spt}(n)=\frac{1}{12} \operatorname{Tr}_{24 n-1,6,1}(g-P) .
$$

By combining (1.3) with spectral methods, we will prove the following asymptotic formula with a power saving error term for $\operatorname{spt}(n)$. 
Theorem 1.3. Let $n \in \mathbb{Z}^{+}$be such that $24 n-1$ is square-free. Then

$$
\begin{gathered}
\operatorname{spt}(n)=\frac{1}{12} \sum_{\tau \in R_{24 n-1,6,1}} e(-\tau)-\frac{1}{12} \sum_{\tau \in R_{24 n-1,6,1}^{*}}\left(1-\frac{1}{2 \pi \operatorname{Im}(\tau)}\right) e(-\tau) \\
+\frac{h(-24 n+1)}{12}\left(\langle g, 1\rangle_{\mathrm{reg}}-\langle P, 1\rangle_{\mathrm{reg}}\right) \\
+\frac{h(-24 n+1)}{4 \pi}\left(\frac{1}{\frac{24}{\pi}+(24 n-1)^{-\frac{1}{240}}}-\frac{12}{(24 n-1)^{\frac{28}{239}}}\right) \\
+\#\left\{\tau \in \mathcal{O}_{24 n-1,6,1}: \frac{(24 n-1)^{\frac{28}{239}}}{12}<\operatorname{Im}(\tau) \leq \frac{\sqrt{24 n-1}}{12}\right\} \\
+O_{\varepsilon}\left(n^{\frac{1}{2}-\frac{1}{2868}+\varepsilon}\right),
\end{gathered}
$$

where

$$
R_{24 n-1,6,1}:=\left\{\tau \in \mathcal{O}_{24 n-1,6,1}: \operatorname{Im}(\tau)>\frac{24}{\pi}+(24 n-1)^{-\frac{1}{240}}\right\} .
$$

Remark 1.4. In Section 5 we plot the Heegner points in $\Lambda_{24 n-1,6}$ for some small values of $n$. Note that $R_{24 n-1,6,1} \subset R_{24 n-1,6,1}^{*}$, and moreover, $R_{24 n-1,6,1}^{*} \neq \varnothing$ if and only if $n \geq 443$, and $R_{24 n-1,6,1} \neq \varnothing$ if and only if $n \geq 444$.

We now discuss the relation of Theorem 1.3 to some existing work. Define the symmetrized second rank moment function

$$
\eta_{2}(n):=\sum_{m \in \mathbb{Z}}\left(\begin{array}{c}
m \\
2
\end{array}\right) N(n, m)
$$

where $N(n, m)$ denotes the number of partitions of $n$ with rank $m$. In $[\mathrm{A}]$, Andrews proved (among many other things) that $\eta_{2}(n)$ equals the number of 2-marked Durfee symbols associated to partitions of $n$ (see Section 1.4). In a subsequent paper, Andrews [A2, Theorem 3] proved that

$$
\operatorname{spt}(n)=n p(n)-\eta_{2}(n)
$$

Consider the generating function

$$
R_{2}(q):=\sum_{n=0}^{\infty} \eta_{2}(n) q^{n}, \quad q:=e^{2 \pi i z} .
$$

Bringmann [B, Theorem 1.1] proved that, up to addition by a certain quasi-modular form, $R_{2}(q)$ is the holomorphic part of a harmonic weak Maass form of weight $3 / 2$ for $\Gamma_{0}(576)$. By combining this result with the circle method, Bringmann [B, Theorem 1.2] gave an asymptotic formula for $\eta_{2}(n)$, or equivalently, the number of 2-marked Durfee symbols associated to partitions of $n$, with an error term which is $O\left(n^{1+\varepsilon}\right)$. Given (1.4) and the known asymptotics for $p(n)$, one immediately deduces an asymptotic formula for $\operatorname{spt}(n)$ with an error term which is $O\left(n^{1+\varepsilon}\right)$. The error term in Theorem 1.3 saves a power of $n$. On the other hand, Bringmann's theorem relating $R_{2}(q)$ to harmonic weak Maass forms is a crucial input to the proof of the Ahlgren-Andersen formula (1.3), which was the starting point of our analysis. 
1.4. Asymptotics for rank moments and Durfee symbols. In their study of partition congruences, Atkin and Garvan [AtG] defined the $k$-th rank moment function

$$
N_{k}(n):=\sum_{m \in \mathbb{Z}} m^{k} N(m, n)
$$

Observe that since $N(m, n) \neq 0$ for only finitely many $m \in \mathbb{Z}$, the series $N_{k}(n)$ is finite. The symmetrized $k$-th rank moment function is defined by

$$
\eta_{k}(n):=\sum_{m \in \mathbb{Z}}\left(\begin{array}{c}
m+\left\lfloor\frac{k-1}{2}\right\rfloor \\
k
\end{array}\right) N(m, n) .
$$

Given a partition $\lambda=\left(\lambda_{1}, \ldots, \lambda_{t}\right)$ of an integer $n$, we say that $\lambda$ has a Durfee square of side length $s$ if $1 \leq s \leq t$ is the largest integer such that the $s$-th part $\lambda_{s} \geq s$. In other words, the largest square contained in the Ferrers graph of $\lambda$ is of size $s \times s$. The Durfee symbol associated to $\lambda$ is an array consisting of two rows and a subscript. The first row consists of the partition obtained by counting the number of nodes in each column to the right of the Durfee square in the Ferrers graph of $\lambda$. The second row consists of the partition below the Durfee square. The subscript is the length of the side of the Durfee square. For example, the following figure illustrates the Durfee square and the Durfee symbol associated to the partition $23=7+5+3+3+3+2$.

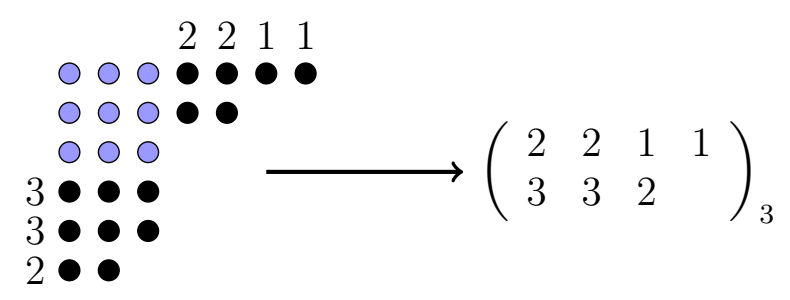

Figure 1. The Durfee square and the Durfee symbol associated to the partition $23=7+5+3+3+3+2$.

Remark 1.5. Let $R_{1}$ and $R_{2}$ denote the first and second rows of the Durfee symbol of $\lambda$, respectively. Then the largest part $\lambda_{1}=s+\# R_{1}$ and the total number of parts $t=s+\# R_{2}$. Hence the rank of $\lambda$ is $\operatorname{rank}(\lambda)=\lambda_{1}-t=\# R_{1}-\# R_{2}$, the number of elements in the first row of the Durfee symbol minus the number of elements in the second row.

Andrews [A] introduced a refinement of the Durfee symbol by "marking" the parts of the partitions appearing in the rows of the Durfee symbol using different copies of the integers. For a positive integer $k$, we designate $k$ different copies of the positive integers by $\mathbb{Z}_{1}^{+}=\left\{1_{1}, 2_{1}, 3_{1}, \ldots\right\}, \mathbb{Z}_{2}^{+}=\left\{1_{2}, 2_{2}, 3_{2}, \ldots\right\}, \ldots, \mathbb{Z}_{k}^{+}=\left\{1_{k}, 2_{k}, 3_{k}, \ldots\right\}$. The $k$-marked Durfee symbols associated to a partition $\lambda$ are then defined as follows.

Starting from the Durfee symbol associated to $\lambda$, we replace the parts in each row by the corresponding parts of the different copies of the integers $\mathbb{Z}_{i}^{+}$according to the following rules.

(1) The sequence of entries and the sequence of subscripts in each row must be nonincreasing.

(2) For each $1 \leq j \leq k-1$, at least one element of $\mathbb{Z}_{j}^{+}$must appear as a part in the first row. 
(3) For each $1 \leq j \leq k-1$, let $M_{j}$ denote the largest element of $\mathbb{Z}_{j}^{+}$appearing as a part in the first row and let $M_{0}:=1$ and $M_{k}:=s$, where $s$ is the length of the side of the Durfee square associated to $\lambda$. Then for $1 \leq i \leq k$, every element of $\mathbb{Z}_{i}^{+}$appearing as a part in the second row must lie in the interval $\left[M_{i-1}, M_{i}\right]$.

A symbol formed from the Durfee symbol associated to $\lambda$ by the above rules is called a $k$-marked Durfee symbol associated to $\lambda$.

For example, there are six 2-marked Durfee symbols associated to the partition $(7,5,3,3,3,2)$ of 23, whose Durfee symbol and Durfee square are displayed in Figure 1. These 2-marked Durfee symbols are given in the following list:

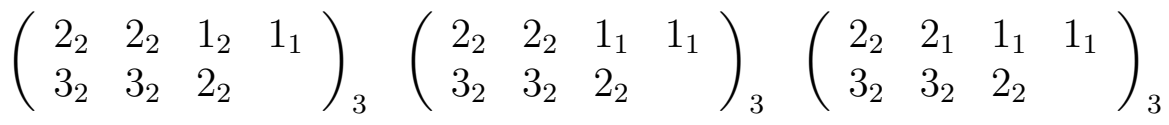

$$
\begin{aligned}
& \left(\begin{array}{llll}
2_{2} & 2_{1} & 1_{1} & 1_{1} \\
3_{2} & 3_{2} & 2_{1} & )_{3}
\end{array}\right)_{3}\left(\begin{array}{llll}
2_{1} & 2_{1} & 1_{1} & 1_{1} \\
3_{2} & 3_{2} & 2_{2} &
\end{array}\right)_{3}\left(\begin{array}{llll}
2_{1} & 2_{1} & 1_{1} & 1_{1} \\
3_{2} & 3_{2} & 2_{1} &
\end{array}\right)_{3}
\end{aligned}
$$

Let $\mathcal{D}_{k}(n)$ denote the number of $k$-marked Durfee symbols associated to partitions of $n$. Andrews [A, Corollary 13] proved that

$$
\eta_{2 k}(n)=\mathcal{D}_{k+1}(n)
$$

thus providing a combinatorial interpretation of the $2 k$-th symmetrized rank moment function.

Now, the identities (1.4) and (1.5) imply that

$$
\mathcal{D}_{2}(n)=n p(n)-\operatorname{spt}(n) .
$$

Hence by combining (1.6), Theorem 1.1 and Theorem 1.3, we get the following asymptotic formula with a power saving error term for $\mathcal{D}_{2}(n)$.

Theorem 1.6. Let $n \in \mathbb{Z}^{+}$be such that $24 n-1$ is square-free. Then

$$
\begin{gathered}
\mathcal{D}_{2}(n)=-\frac{1}{12} \sum_{\tau \in R_{24 n-1,6,1}} e(-\tau)+\frac{c(n)}{12} \sum_{\tau \in R_{24 n-1,6,1}^{*}}\left(1-\frac{1}{2 \pi \operatorname{Im}(\tau)}\right) e(-\tau) \\
+\frac{h(-24 n+1)}{12}\left(c(n)\langle P, 1\rangle_{\mathrm{reg}}-\langle g, 1\rangle_{\mathrm{reg}}\right) \\
-\frac{h(-24 n+1)}{4 \pi}\left(\frac{1}{\frac{24}{\pi}+(24 n-1)^{-\frac{1}{240}}}-\frac{12}{(24 n-1)^{\frac{28}{239}}}\right) \\
-\#\left\{\tau \in \mathcal{O}_{24 n-1,6,1}: \frac{(24 n-1)^{\frac{28}{239}}}{12}<\operatorname{Im}(\tau) \leq \frac{\sqrt{24 n-1}}{12}\right\} \\
+O_{\varepsilon}\left(n^{\frac{1}{2}-\frac{1}{2868}+\varepsilon}\right),
\end{gathered}
$$

where

$$
c(n):=\frac{36 n-1}{24 n-1}
$$


1.5. Acknowledgments. We would like to thank Sheng-Chi Liu for helpful discussions regarding this work. The authors were supported in part by the NSF grants DMS-1162535 (R.M.) and DMS-1460766 (Texas A\&M U. mathematics REU). In addition, the second author was supported in part by the University of Costa Rica.

\section{TRACES OF WEAKLY HOLOMORPHIC FORMS}

Let $f \in M_{0}^{!}(N)$ be a weakly holomorphic form of weight zero for $\Gamma_{0}(N)$. Such a form $f(z)$ has a Fourier expansion in the cusp at infinity given by

$$
f(z)=\sum_{n=0}^{M} a(-n) e(-n z)+\sum_{n=1}^{\infty} a(n) e(n z)
$$

for some integer $M \geq 0$.

The following asymptotic formula for the trace of $f(z)$ was proved by the third author in [M2, Theorem 1.1].

Theorem 2.1 ([M2], Theorem 1.1). We have

$$
\operatorname{Tr}_{D, N, h}(f)=\sum_{n=0}^{M} a(-n) \sum_{\tau \in R_{D, N, h}} e(-n \tau)+h(-D)\langle f, 1\rangle_{\mathrm{reg}}+O_{N, \varepsilon}\left(D^{\frac{1}{2}-\frac{1}{240}+\varepsilon}\right) .
$$

The first few terms in the Fourier expansion at infinity of the weakly holomorphic form $g \in M_{0}^{!}(6)$ defined by $(1.2)$ are

$$
g(z)=e(-z)+12+77 \cdot e(z)+\cdots .
$$

Given (2.1), Theorem 2.1 immediately implies the following result.

Proposition 2.2. Let $n \in \mathbb{Z}^{+}$be such that $24 n-1$ is square-free. Then

$$
\operatorname{Tr}_{24 n-1,6,1}(g)=\sum_{\tau \in R_{24 n-1,6,1}} e(-\tau)+h(-24 n+1)\langle g, 1\rangle_{\mathrm{reg}}+12 \cdot \# R_{24 n-1,6,1}+O_{\varepsilon}\left(n^{\frac{1}{2}-\frac{1}{240}+\varepsilon}\right) .
$$

Remark 2.3. The classical modular $j$-function

$$
j(z)=e(-z)+744+196884 \cdot e(z)+\cdots
$$

is a weakly holomorphic modular form of weight zero for $\mathrm{SL}_{2}(\mathbb{Z})$. The values of $j(z)$ at Heegner points are algebraic integers called singular moduli. Zagier [Za] proved that traces of singular moduli are Fourier coefficients of a weight $3 / 2$ modular form for $\Gamma_{0}(4)$ in Kohnen's plus space. Bruinier, Jenkins and Ono [BJO] studied the asymptotic distribution of these traces and conjectured the precise form of the limiting distribution. This conjecture was proved by Duke in $[\mathrm{D}]$.

\section{Counting Heegner points in An expanding Rectangle}

The appearance of $\# R_{24 n-1,6,1}$ in Proposition 2.2 leads us naturally to the problem of counting Heegner points in an "expanding" rectangle.

We will need the following lemma.

Lemma 3.1. Let $\bar{\gamma} \in \Gamma_{\infty} \backslash \Gamma_{0}(N)$ with $\bar{\gamma} \neq \bar{I}$. Then given a Heegner point $\tau \in \mathcal{O}_{D, N, h}$, we have

$$
\operatorname{Im}(\gamma \tau) \leq \frac{4 N}{\pi}
$$


Proof. If $\bar{\gamma} \in \Gamma_{\infty} \backslash \Gamma_{0}(N)$ with $\bar{\gamma} \neq \bar{I}$, then

$$
\gamma=\left(\begin{array}{ll}
a & b \\
c & d
\end{array}\right) \in \mathrm{SL}_{2}(\mathbb{Z})
$$

with $c \neq 0$ (hence $c^{2} \geq 1$ ). Recall that $\tau \in \mathcal{O}_{D, N, h}$ has the form

with

$$
\tau=\tau_{\mathfrak{A}}^{(h)}=\frac{b+\sqrt{-D}}{2 N a}
$$

$$
a=N(\mathfrak{A}) \leq \frac{2}{\pi} \sqrt{D}
$$

Now, we have

$$
\operatorname{Im}(\gamma \tau)=\frac{\operatorname{Im}(\tau)}{|c \tau+d|^{2}}=\frac{1}{|c \tau+d|^{2}} \frac{\sqrt{D}}{2 N a}
$$

Since $c^{2} \geq 1$, we get

$$
|c \tau+d|^{2}=\left(\frac{c b}{2 N a}+d\right)^{2}+\left(\frac{c \sqrt{D}}{2 N a}\right)^{2} \geq \frac{D}{4 N^{2} a^{2}} .
$$

Then (3.1) implies that

$$
\operatorname{Im}(\gamma \tau) \leq \frac{4 N^{2} a^{2}}{D} \frac{\sqrt{D}}{2 N a}=\frac{2 N a}{\sqrt{D}} \leq \frac{4 N}{\pi}
$$

Let $L\left(\chi_{-D}, s\right)$ be the Dirichlet $L$-function associated to the Kronecker symbol $\chi_{-D}$.

Proposition 3.2. For $b>0$ and $0<\delta<1 / 2$, define

Assume that

$$
R_{D, N, h, b, \delta}:=\left\{\tau \in \mathcal{O}_{D, N, h}: \frac{4 N}{\pi}+D^{-b} \leq \operatorname{Im}(\tau) \leq \frac{D^{\frac{1}{2}-\delta}}{2 N}\right\} .
$$

$$
L\left(\chi_{-D}, \frac{1}{2}+i t\right) \ll_{\varepsilon}(1+|t|)^{B+\varepsilon} D^{\frac{1}{4}-\delta_{1}+\varepsilon}
$$

for some $0 \leq B<1$ and $0<\delta_{1} \leq 1 / 4$. Then for any $\delta_{2}>b$, we have

$$
\begin{gathered}
\# R_{D, N, h, b, \delta}=\frac{h(-D)}{\operatorname{vol}\left(Y_{0}(N)\right)}\left(\frac{1}{\frac{4 N}{\pi}+D^{-b}}-\frac{2 N}{D^{\frac{1}{2}-\delta}}\right) \\
\quad+O_{N, \varepsilon}\left(D^{\frac{1}{2}-b+\varepsilon}\right)+O_{N, \varepsilon}\left(D^{\frac{1}{2}-\left(\delta_{1}-b\right)+\varepsilon}\right) \\
\quad+O_{N, \varepsilon}\left(D^{\frac{1}{2}-f_{1}\left(\delta, \delta_{1}, b\right)+\varepsilon}\right)+O_{N, B, \varepsilon}\left(D^{\frac{1}{2}-f_{2}\left(\delta, \delta_{1}, \delta_{2}, B\right)+\varepsilon}\right),
\end{gathered}
$$

where

$$
\begin{aligned}
f_{1}\left(\delta, \delta_{1}, b\right) & :=\frac{\delta}{2}+\left(\delta_{1}-\frac{1}{4}\right)-b \\
f_{2}\left(\delta, \delta_{1}, \delta_{2}, B\right) & :=\left(B+\frac{1}{2}\right) \delta+\left(\delta_{1}-\frac{1}{4}\right)-(B+1) \delta_{2}-\frac{B}{2}
\end{aligned}
$$


Remark 3.3. The asymptotic formula in Proposition 3.2 is meaningful for any $b, \delta$ satisfying the inequalities

$0<b<\delta_{1}, \quad 2\left(b+\left(\frac{1}{4}-\delta_{1}\right)\right)<\delta<\frac{1}{2}, \quad \frac{1}{B+\frac{1}{2}}\left(\left(\frac{1}{4}-\delta_{1}\right)+(B+1) \delta_{2}+\frac{B}{2}\right)<\delta<\frac{1}{2}$.

There exists a $\delta$ satisfying the second and third inequalities if

$$
2\left(b+\left(\frac{1}{4}-\delta_{1}\right)\right)<\frac{1}{2} \Longleftrightarrow b<\delta_{1}
$$

and

$$
\frac{1}{B+\frac{1}{2}}\left(\left(\frac{1}{4}-\delta_{1}\right)+(B+1) \delta_{2}+\frac{B}{2}\right)<\frac{1}{2} \Longleftrightarrow \delta_{2}<\frac{\delta_{1}}{B+1} .
$$

The Lindelöf hypothesis implies that (3.2) holds with $B=0$ and $\delta_{1}=1 / 4$. In this case, the asymptotic formula is meaningful for any $b, \delta$ such that $0<b<1 / 4$ and $2 \delta_{2}<\delta<1 / 2$ where $b<\delta_{2}<1 / 4$. Recently, M. Young [Y] proved that (3.2) holds with $B=1 / 6$ and $\delta_{1}=1 / 12$, i.e., Weyl-subconvexity in both the $t$ and $D$-aspects (see [Y, Theorem 1.1, eqn. (1.8)] and the discussion following [Y, Theorem 1.1]). In this case, the asymptotic formula is meaningful for any $b, \delta$ such that $0<b<1 / 12$ and $\max \left\{2 b+\frac{1}{3}, \frac{3}{8}+\frac{7}{4} \delta_{2}\right\}<\delta<1 / 2$ where $b<\delta_{2}<1 / 14$.

Proof of Proposition 3.2: Let $\lambda_{1}, \lambda_{2}: \mathbb{R} \rightarrow[0,1]$ be $C^{\infty}$ functions such that

$$
\lambda_{1}(t)=\left\{\begin{array}{ll}
0, & t \leq 0 \\
1, & t \geq 1
\end{array}, \quad \lambda_{2}(t)= \begin{cases}1, & t \leq 0 \\
0, & t \geq 1\end{cases}\right.
$$

Define the functions

$$
\phi_{1}(t):=\lambda_{1}\left(\frac{t-\frac{4 N}{\pi}}{D^{-b}}\right), \quad \phi_{2}(t):=\lambda_{2}\left(\frac{t-\frac{D^{1 / 2-\delta}}{2 N}}{D^{-b}}\right), \quad \phi(t)=\phi_{D, N, b, \delta}(t):=\phi_{1}(t) \phi_{2}(t) .
$$

Then $\phi: \mathbb{R} \rightarrow[0,1]$ is a $C^{\infty}$ function which is supported on $\left(\frac{4 N}{\pi}, \frac{D^{1 / 2-\delta}}{2 N}+D^{-b}\right)$, equals 1 on $\left[\frac{4 N}{\pi}+D^{-b}, \frac{D^{1 / 2-\delta}}{2 N}\right]$, and satisfies the bound

$$
\phi^{(A)} \ll D^{A b}, \quad A=0,1,2, \ldots
$$

where the implied constant is independent of $D, N$ and $\delta$.

To ease notation, we set $R_{b, \delta}:=R_{D, N, h, b, \delta}$. Define the incomplete Eisenstein series

$$
g_{\phi}(z):=\sum_{\bar{\gamma} \in \Gamma_{\infty} \backslash \Gamma_{0}(N)} \phi(\operatorname{Im}(\gamma z))
$$

Then by Lemma 3.1, we have

$$
\begin{aligned}
\sum_{\tau \in \mathcal{O}_{D, N, h}} g_{\phi}(\tau) & =\sum_{\tau \in \mathcal{O}_{D, N, h}} \sum_{\substack{\gamma \in \Gamma_{\infty} \backslash \Gamma_{0}(N) \\
\bar{\gamma} \neq \bar{I}}} \phi(\operatorname{Im}(\gamma \tau))+\sum_{\tau \in \mathcal{O}_{D, N, h}} \phi(\operatorname{Im}(\tau)) \\
& =\# R_{b, \delta}+\sum_{\tau \notin R_{b, \delta}} \phi(\operatorname{Im}(\tau)) .
\end{aligned}
$$


The real-analytic Eisenstein series

$$
E_{\infty}(z, s):=\sum_{\bar{\gamma} \in \Gamma_{\infty} \backslash \Gamma_{0}(N)} \operatorname{Im}(\gamma z)^{s}, \quad \operatorname{Re}(s)>1
$$

has a meromorphic continuation to $\mathbb{C}$ with a simple pole at $s=1$ with residue $1 / \operatorname{vol}\left(Y_{0}(N)\right)$ (see [I, Theorem 11.3 and Proposition 6.13]). Then by [I, (7.12)], we have

$$
g_{\phi}(z)=\frac{1}{\operatorname{vol}\left(Y_{0}(N)\right)} \widehat{\phi}(1)+\frac{1}{2 \pi i} \int_{\mathbb{R}} \widehat{\phi}\left(\frac{1}{2}+i t\right) E_{\infty}\left(z, \frac{1}{2}+i t\right) d t
$$

where

$$
\widehat{\phi}(s):=\int_{0}^{\infty} \phi(u) u^{-(s+1)} d u
$$

is the Mellin transform of $\phi$. Sum over the Heegner points $\tau \in \mathcal{O}_{D, N, h}$ to get

$$
\sum_{\tau \in \mathcal{O}_{D, N, h}} g_{\phi}(\tau)=\frac{h(-D)}{\operatorname{vol}\left(Y_{0}(N)\right)} \widehat{\phi}(1)+\frac{1}{2 \pi i} \int_{\mathbb{R}} \widehat{\phi}\left(\frac{1}{2}+i t\right) W_{\infty}(D, t) d t
$$

where

$$
W_{\infty}(D, t):=\sum_{\tau \in \mathcal{O}_{D, N, h}} E_{\infty}\left(\tau, \frac{1}{2}+i t\right) .
$$

A straightforward calculation yields

$$
\widehat{\phi}(1)=\frac{1}{\frac{4 N}{\pi}+D^{-b}}-\frac{2 N}{D^{1 / 2-\delta}}+O\left(D^{-b}\right) .
$$

We will estimate

$$
\frac{1}{2 \pi i} \int_{\mathbb{R}} \widehat{\phi}\left(\frac{1}{2}+i t\right) W_{\infty}(D, t) d t
$$

by dividing the integral over $\mathbb{R}$ into two regions.

First, we integrate by parts $A$-times and use the bound (3.3) to get

$$
\widehat{\phi}\left(\frac{1}{2}+i t\right) \ll_{N} D^{A b} D^{-\frac{1}{4}+\frac{\delta}{2}}\left(\frac{D^{\frac{1}{2}-\delta}}{1+|t|}\right)^{A} .
$$

Next, we combine (3.2) with an argument similar to that in [M2, Proposition 2.1] to get

$$
W_{\infty}(D, t) \ll_{\varepsilon}(1+|t|)^{B+\varepsilon} D^{\frac{1}{2}-\delta_{1}+\varepsilon}
$$

for some $0 \leq B<1$ and $0<\delta_{1} \leq 1 / 4$.

Now, fix $\delta_{2}>b$, and divide the integral over $\mathbb{R}$ into the regions $|t| \gg D^{\frac{1}{2}-\delta+\delta_{2}}$ and $|t| \ll D^{\frac{1}{2}-\delta+\delta_{2}}$.

Since $A$ can be chosen to be arbitrarily large, by applying (3.6) and (3.7) in the first region, we get

$$
\mathrm{I}:=\frac{1}{2 \pi i} \int_{|t| \gg D^{\frac{1}{2}-\delta+\delta_{2}}} \widehat{\phi}\left(\frac{1}{2}+i t\right) W_{\infty}(D, t) d t \ll_{N, B, \varepsilon} D^{-C}
$$

for any $C>0$ (here we used $\delta_{2}>b$ ). 
On the other hand, by applying (3.6) (with $A=0$ ) and (3.7) in the second region, we get

$$
\mathrm{II}:=\frac{1}{2 \pi i} \int_{|t| \ll D^{\frac{1}{2}-\delta+\delta_{2}}} \widehat{\phi}\left(\frac{1}{2}+i t\right) W_{\infty}(D, t) d t \ll_{N, B, \varepsilon} D^{\frac{1}{2}-f_{2}\left(\delta, \delta_{1}, \delta_{2}, B\right)+\varepsilon},
$$

where

$$
f_{2}\left(\delta, \delta_{1}, \delta_{2}, B\right):=\left(B+\frac{1}{2}\right) \delta+\left(\delta_{1}-\frac{1}{4}\right)-(B+1) \delta_{2}-\frac{B}{2} .
$$

Combining the estimates for I and II yields

$$
\frac{1}{2 \pi i} \int_{\mathbb{R}} \widehat{\phi}\left(\frac{1}{2}+i t\right) W_{\infty}(D, t) d t=O_{N, B, \varepsilon}\left(D^{\frac{1}{2}-f_{2}\left(\delta, \delta_{1}, \delta_{2}, B\right)+\varepsilon}\right) .
$$

Using (3.5), (3.8), and the bound

$$
h(-D) \ll_{\varepsilon} D^{\frac{1}{2}+\varepsilon},
$$

we get

$$
\begin{aligned}
\sum_{\tau \in \mathcal{O}_{D, N, h}} g_{\phi}(\tau)= & \frac{h(-D)}{\operatorname{vol}\left(Y_{0}(N)\right)}\left(\frac{1}{\frac{4 N}{\pi}+D^{-b}}-\frac{2 N}{D^{1 / 2-\delta}}\right) \\
& +O_{N, \varepsilon}\left(D^{\frac{1}{2}-b+\varepsilon}\right)+O_{N, B, \varepsilon}\left(D^{\frac{1}{2}-f_{2}\left(\delta, \delta_{1}, \delta_{2}, B\right)+\varepsilon}\right) .
\end{aligned}
$$

From (3.4), we see that it remains to estimate the contribution of

$$
\sum_{\tau \notin R_{b, \delta}} \phi(\operatorname{Im}(\tau))
$$

Observe that

$$
\begin{aligned}
\sum_{\tau \notin R_{b, \delta}} \phi(\operatorname{Im}(\tau))= & \sum_{\frac{4 N}{\pi} \leq \operatorname{Im}(\tau)<\frac{4 N}{\pi}+D^{-b}} \phi(\operatorname{Im}(\tau))+\sum_{\frac{D^{1 / 2-\delta}}{2 N}<\operatorname{Im}(\tau) \leq \frac{D^{1 / 2-\delta}}{2 N}+D^{-b}} \phi(\operatorname{Im}(\tau)) \\
\leq \# & \left\{\tau \in \mathcal{O}_{D, N, h}: \frac{4 N}{\pi} \leq \operatorname{Im}(\tau)<\frac{4 N}{\pi}+D^{-b}\right\} \\
& +\#\left\{\tau \in \mathcal{O}_{D, N, h}: \frac{D^{1 / 2-\delta}}{2 N}<\operatorname{Im}(\tau) \leq \frac{D^{1 / 2-\delta}}{2 N}+D^{-b}\right\} \\
= & : R_{b}^{\prime}+R_{b, \delta}^{\prime \prime} .
\end{aligned}
$$

The numbers $R_{b}^{\prime}$ and $R_{b, \delta}^{\prime \prime}$ can be estimated using a modification of the proof of (3.10). We sketch the estimate of $R_{b, \delta}^{\prime \prime}$, leaving the (simpler) estimate of $R_{b}^{\prime}$ to the reader.

Let $\psi: \mathbb{R} \rightarrow[0,1]$ be a $C^{\infty}$ function which is supported on $\left(\frac{D^{1 / 2-\delta}}{2 N}-D^{-b}, \frac{D^{1 / 2-\delta}}{2 N}+2 D^{-b}\right)$, equals 1 on $\left[\frac{D^{1 / 2-\delta}}{2 N}, \frac{D^{1 / 2-\delta}}{2 N}+D^{-b}\right]$, and satisfies the bound

$$
\psi^{(A)} \ll D^{A b}, \quad A=0,1,2, \ldots
$$

where the implied constant is independent of $D, N$ and $\delta$.

Define the incomplete Eisenstein series

$$
g_{\psi}(z):=\sum_{\bar{\gamma} \in \Gamma_{\infty} \backslash \Gamma_{0}(N)} \psi(\operatorname{Im}(\gamma z))
$$


A minor variant of (3.4) shows that

$$
\# R_{b, \delta}^{\prime \prime} \leq \sum_{\tau \in \mathcal{O}_{D, N, h}} g_{\psi}(\tau)
$$

Again, we have

$$
\sum_{\tau \in \mathcal{O}_{D, N, h}} g_{\psi}(\tau)=\frac{h(-D)}{\operatorname{vol}\left(Y_{0}(N)\right)} \widehat{\psi}(1)+\frac{1}{2 \pi i} \int_{\mathbb{R}} \widehat{\psi}\left(\frac{1}{2}+i t\right) W_{\infty}(D, t) d t
$$

where $\widehat{\psi}$ is the Mellin transform of $\psi$.

A straightforward estimate yields

$$
\widehat{\psi}(1) \ll D^{-b}
$$

Now, integrate by parts $A$-times, then use (3.12) and the expansion

$$
(1+X)^{\alpha}=1+\alpha X+O_{\alpha}\left(X^{2}\right), \quad|X|<1, \quad \alpha \in \mathbb{R}
$$

to get

$$
\widehat{\psi}\left(\frac{1}{2}+i t\right) \ll_{A, N} \frac{D^{(A-1) b}}{(1+|t|)^{A}}\left(D^{\frac{1}{2}-\delta}\right)^{A-\frac{3}{2}} .
$$

Then using (3.14) (with $A=2$ ) and (3.7), we get

$$
\frac{1}{2 \pi i} \int_{\mathbb{R}} \widehat{\psi}\left(\frac{1}{2}+i t\right) W_{\infty}(D, t) d t=O_{N, \varepsilon}\left(D^{\frac{1}{2}-f_{1}\left(\delta, \delta_{1}, b\right)+\varepsilon}\right),
$$

where

$$
f_{1}\left(\delta, \delta_{1}, b\right):=\frac{\delta}{2}+\left(\delta_{1}-\frac{1}{4}\right)-b .
$$

From (3.13), (3.15) and (3.9), we get

$$
\# R_{b, \delta}^{\prime \prime}=O_{N, \varepsilon}\left(D^{\frac{1}{2}-b+\varepsilon}\right)+O_{N, \varepsilon}\left(D^{\frac{1}{2}-f_{1}\left(\delta, \delta_{1}, b\right)+\varepsilon}\right) .
$$

A similar argument shows that

$$
\# R_{b}^{\prime}=O_{N, \varepsilon}\left(D^{\frac{1}{2}-b+\varepsilon}\right)+O_{N, \varepsilon}\left(D^{\frac{1}{2}-\left(\delta_{1}-b\right)+\varepsilon}\right) .
$$

Finally, by combining (3.4), (3.10), (3.11), (3.16) and (3.17), for $\delta_{2}>b$ we get

$$
\begin{gathered}
\# R_{b, \delta}=\frac{h(-D)}{\operatorname{vol}\left(Y_{0}(N)\right)}\left(\frac{1}{\frac{4 N}{\pi}+D^{-b}}-\frac{2 N}{D^{1 / 2-\delta}}\right) \\
\quad+O_{N, \varepsilon}\left(D^{\frac{1}{2}-b+\varepsilon}\right)+O_{N, \varepsilon}\left(D^{\frac{1}{2}-\left(\delta_{1}-2 b\right)+\varepsilon}\right) \\
\quad+O_{N, \varepsilon}\left(D^{\frac{1}{2}-f_{1}\left(\delta, \delta_{1}, b\right)+\varepsilon}\right)+O_{N, B, \varepsilon}\left(D^{\frac{1}{2}-f_{2}\left(\delta, \delta_{1}, \delta_{2}, B\right)+\varepsilon}\right) .
\end{gathered}
$$




\section{Proof of Theorem 1.3}

Combine (1.1) and (1.3) to get

$$
\operatorname{spt}(n)=\frac{1}{12} \operatorname{Tr}_{24 n+1,6,1}(g)-\frac{24 n-1}{12} p(n) .
$$

Now, by Proposition 2.2 and Theorem 1.1, we have

$$
\begin{gathered}
\operatorname{spt}(n)=\frac{1}{12} \sum_{\tau \in R_{24 n-1,6,1}} e(-\tau)-\frac{1}{12} \sum_{\tau \in R_{24 n-1,6,1}^{*}}\left(1-\frac{1}{2 \pi \operatorname{Im}(\tau)}\right) e(-\tau) \\
+\frac{h(-24 n+1)}{12}\left(\langle g, 1\rangle_{\mathrm{reg}}-\langle P, 1\rangle_{\mathrm{reg}}\right)+\# R_{24 n-1,6,1} \\
+O_{\varepsilon}\left(n^{\frac{1}{2}-\frac{1}{240}+\varepsilon}\right) .
\end{gathered}
$$

On the other hand, by Proposition 3.2 with the choices $B=1 / 6, \delta_{1}=1 / 12, b=1 / 240, \delta_{2}=$ $1 / 239$ and $\delta=183 / 478$, we get

$$
\# R_{24 n-1,6,1, \frac{1}{240}, \frac{183}{478}}=\frac{h(-24 n+1)}{4 \pi}\left(\frac{1}{\frac{24}{\pi}+(24 n-1)^{-\frac{1}{240}}}-\frac{12}{(24 n-1)^{\frac{28}{239}}}\right)+O_{\varepsilon}\left(n^{\frac{1}{2}-\frac{1}{2868}+\varepsilon}\right),
$$

where we used $\operatorname{vol}\left(Y_{0}(6)\right)=4 \pi$ (see Remark 4.1 for an explanation of these choices of parameters).

Since

$\# R_{24 n-1,6,1}=\# R_{24 n-1,6,1, \frac{1}{240}, \frac{183}{478}}+\#\left\{\tau \in \mathcal{O}_{24 n-1,6,1}: \frac{(24 n-1)^{\frac{28}{239}}}{12}<\operatorname{Im}(\tau) \leq \frac{\sqrt{24 n-1}}{12}\right\}$

the result follows by combining (4.1) and (4.2).

Remark 4.1. To get (4.2), we inserted the best unconditional constants $B=1 / 6$ and $\delta_{1}=1 / 12$ due to M. Young [Y]. Then given $b=1 / 240$, we chose $\delta_{2}$ so that $\delta$ could be made "small" (see Remark 3.3). Of course, we could choose $\delta_{2}$ and $\delta$ so that the exponent in the error term is smaller, at the expense of increasing the size of $\delta$.

\section{HeEgner POINTS OF SMALL Discriminant}

We used SageMath to plot the Heegner points in $\Lambda_{24 n-1,6}$ on a fundamental domain for $\Gamma_{0}(6)$ for some small values of $n$. Figures 2 and 3 display the Heegner points in $\Lambda_{359,6}$ and $\mathcal{O}_{359,6,1}$, respectively. 


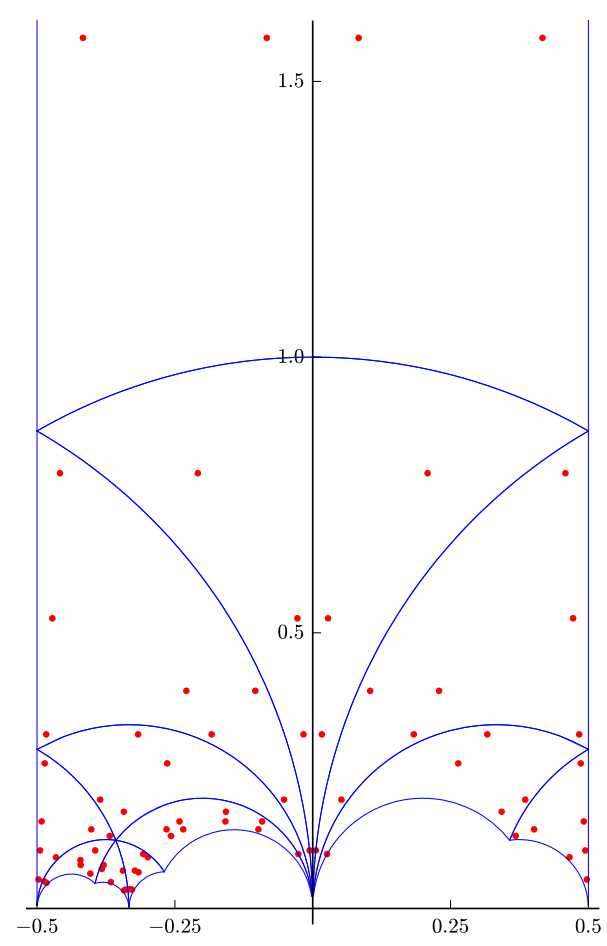

FiguRE 2. Heegner points in $\Lambda_{359,6}$.

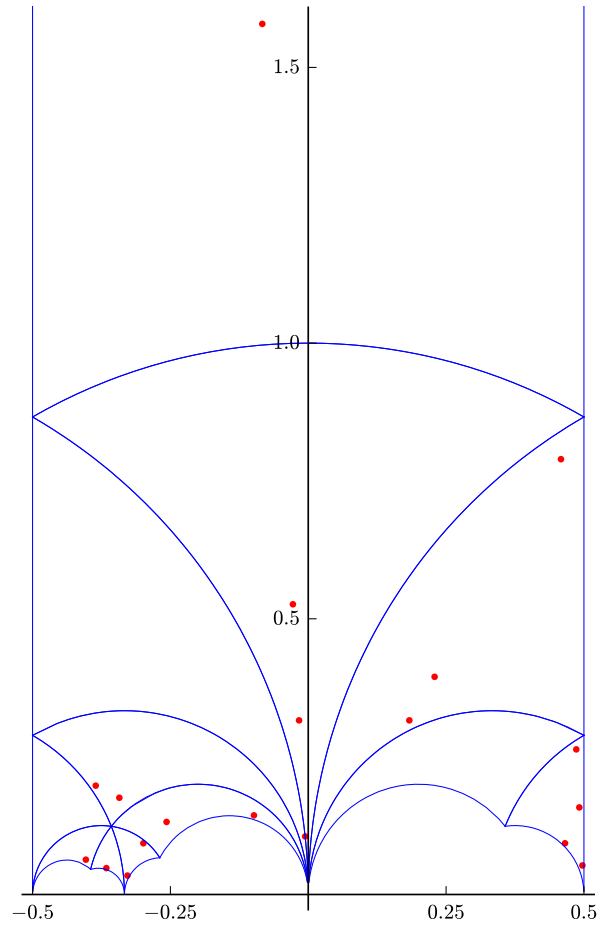

Figure 3. Heegner points in $\mathcal{O}_{359,6,1}$.

Figure 4 displays the Heegner points $\tau \in \Lambda_{119999,6}$ with $\operatorname{Im}(\tau)>\frac{24}{\pi}+(119999)^{-\frac{1}{176}}$, where the points $\tau \in R_{119999,6,1}^{*}$ appear as circles (these are the points in Theorems 1.3 and 1.6 when $n=5000)$.

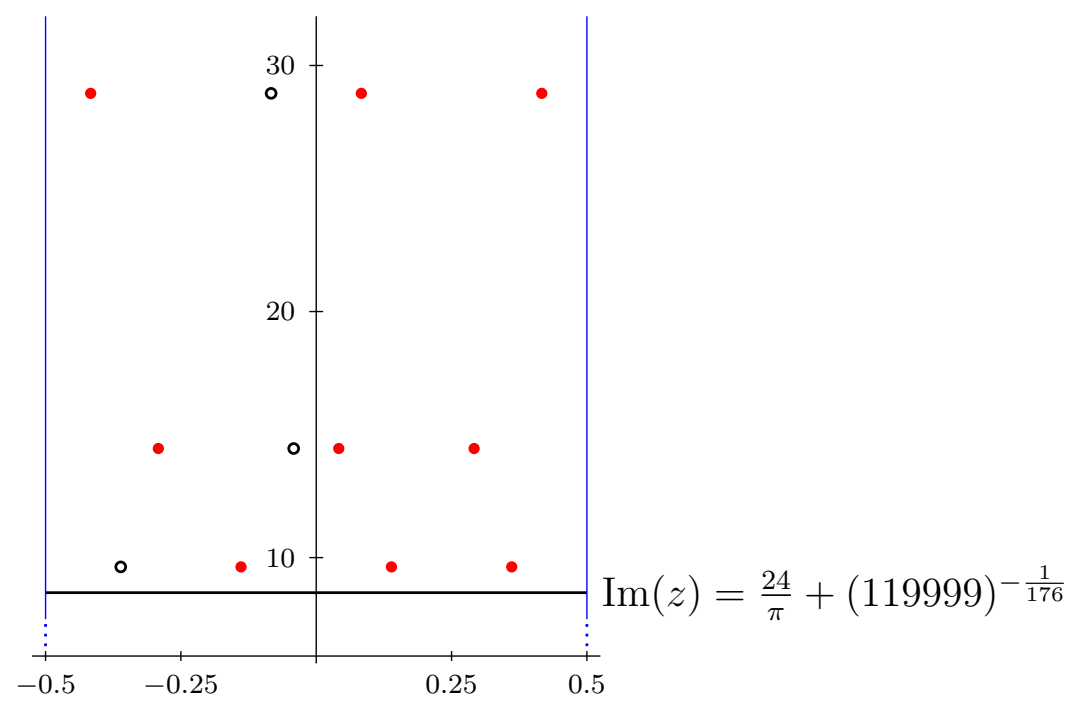

FiguRE 4. Heegner points $\tau \in \Lambda_{119999,6}$ with $\operatorname{Im}(\tau)>\frac{24}{\pi}+(119999)^{-\frac{1}{176}}$. 


\section{REFERENCES}

[AA] S. Ahlgren and N. Andersen, Algebraic and transcendental formulas for the smallest parts function. Preprint available at: arXiv: 1504.02500

[ABL] S. Ahlgren, K. Bringmann and J. Lovejoy, $\ell$-adic properties of smallest parts functions. Adv. Math. 228 (2011), 629-645.

[A] G. E. Andrews, Partitions, Durfee symbols, and the Atkin-Garvan moments of ranks. Invent. Math. 169 (2007), 37-73.

[A2] G. E. Andrews, The number of smallest parts in the partitions of n. J. Reine Angew. Math. 624 (2008), $133-142$.

[AtG] A. O. L. Atkin and F. Garvan, Relations between the ranks and cranks of partitions. Ramanujan J. 7 (2003), 343-366.

[B] K. Bringmann, On the explicit construction of higher deformations of partition statistics. Duke Math. J. 144 (2008), 195-233.

[BrO] K. Bringmann and K. Ono, An arithmetic formula for the partition function. Proc. Amer. Math. Soc. 135 (2007), 3507-3514.

[BJO] J. H. Bruinier, P. Jenkins, and K. Ono, Hilbert class polynomials and traces of singular moduli. Math. Ann. 334 (2006), 373-393.

[BO] J. H. Bruinier and K. Ono, Algebraic formulas for the coefficients of half-integral weight harmonic weak Maass forms. Adv. Math. 246 (2013), 198-219.

[D] W. Duke, Modular functions and the uniform distribution of CM points. Math. Ann. 334 (2006), 241252.

[FM] A. Folsom and R. Masri, Equidistribution of Heegner points and the partition function. Math. Ann. 348 (2010), 289-317.

[FO] A. Folsom and K. Ono, The spt-function of Andrews. Proc. Natl. Acad. Sci. USA 105 (2008), 2015220156.

[G] F. Garvan, Congruences for Andrews' smallest parts partition function and new congruences for Dyson's rank. Int. J. Number Theory 6 (2010) 1-29.

[GZ] B. Gross and D. Zagier, Heegner points and derivatives of L-series. Invent. Math. 84 (1986), 225-320.

[I] H. Iwaniec, Introduction to the spectral theory of automorphic forms. Biblioteca de la Revista Matemática Iberoamericana. Revista Matematica Iberoamericana, Madrid, 1995. xiv+247 pp.

[I2] H. Iwaniec, Topics in classical automorphic forms. Graduate Studies in Mathematics, 17. American Mathematical Society, Providence, RI, 1997. xii+259 pp.

[M] R. Masri, Fourier coefficients of harmonic weak Maass forms and the partition function. American Journal of Mathematics 137 (2015), 1061-1097.

[M2] R. Masri, Singular moduli and the distribution of partition ranks modulo 2. Mathematical Proceedings of the Cambridge Philosophical Society, to appear.

[O] K. Ono, Congruences for the Andrews spt function. Proc. Natl. Acad. Sci. USA 108 (2011), 473-476.

$[\mathrm{Y}]$ M. P. Young, Weyl-type hybrid subconvexity bounds for twisted L-functions and Heegner points on shrinking sets. Journal of the European Mathematical Society, to appear. Preprint available at: arXiv: $1405.5457 \mathrm{v} 2$

[Za] D. Zagier, Traces of singular moduli. Motives, polylogarithms and Hodge theory, Part I (Irvine, CA, 1998), 211-244, Int. Press Lect. Ser., 3, I, Int. Press, Somerville, MA, 2002.

\footnotetext{
Department of Mathematics and Statistics, Youngstown State University, Youngstown, $\mathrm{OH} 44555$

E-mail address: jmbanks01@student.ysu.edu
}

Department of Mathematics, Mailstop 3368, Texas A\&M University, College Station, TX 77843-3368

E-mail address: adrianbs11@math.tamu.edu

E-mail address: masri@math.tamu.edu 
Mathematics \& Computer Science, Mail Stop: 1131-002-1AC, Emory University, Atlanta, GA 30322

E-mail address: kayysheng@gmail.com 